\title{
Local Shape Representation in 3D: from Weighted Spherical Harmonics to Spherical Wavelets
}

Cheng-Jin $\mathrm{Du}^{1}$

c.du@warwick.ac.uk

John G. Ferguson ${ }^{2}$

john.ferguson@bbsrc.ac.uk

Phillip T. Hawkins ${ }^{2}$

phillip.hawkins@bbsrc.ac.uk

Len R. Stephens ${ }^{2}$

len.stephens@bbsrc.ac.uk

Till Bretschneider ${ }^{1}$

T.Bretschneider@warwick.ac.uk

\author{
${ }^{1}$ Warwick Systems Biology Centre, \\ University of Warwick, \\ Coventry, \\ CV4 7AL, \\ UK \\ ${ }^{2}$ The Babraham Institute, \\ Cambridge, \\ CB22 3AT, \\ UK
}

\begin{abstract}
Both weighted spherical harmonics (SPHARM) and spherical wavelets (SW) have been used for local shape representation of biological structures. In this paper, we shed light on the underlying connections between weighted SPHARM and SW. A novel derivation of SW from weighted SPHARM and a detailed formulation of wavelet transformation for shape representation are presented. Their performances on local shape representation are evaluated on both macro (amygdala) and micro (neutrophil cell) scale images. The experimental results show that SW outperforms the weighted SPHARM for local shape representation of large and complex cell images, while the weighted SPHARM is a good choice for representing small and simpler amygdala.
\end{abstract}

\section{Introduction}

Recent advances in imaging techniques have made it possible to acquire high quality 3D volumetric images of biological structures by using a variety of powerful tools, such as magnetic resonance imaging and spinning disk confocal microscopy. Although the acquired images span the spectrum from macro scales (e.g. brain substructures) to micro scales (e.g. cell images), the challenge of extracting fundamental features of biological structures is similar. By comparing images from different individuals or subsequent time points, structural changes can be identified, which are important for studying health and disease. Comparison of biological structures requires effective shape representation, which is inherently difficult because of their considerable natural variability.

Numerous techniques have been proposed for shape representation, including landmarks [1], medial representation [2], and spherical harmonics (SPHARM) [3]. Both the landmarks and the medial representations are discrete representations. A continuum approach, SPHARM was first used as a type of parametric surface representation for starshaped objects [4], and later extended by Brechbul : 
arbitrarily shaped but simply connected objects. One of the advantages of SPHARM is that no landmarks are required for shape representation. Furthermore, the mathematical representation of the 3D shape with an orthogonal basis enables SPHARM to produce a phenotypic space of biological structures, and thus provides a basis for reconstructing evolutionary changes [5]. However, the SPHARM representation suffers from the Gibbs phenomenon [6], where poor results are obtained for rapidly changing or discontinuous data [7]. In addition, it has limited scalability, and is slow when applied to large scale 3D surfaces. Therefore, it has been primarily used to represent small or moderate surfaces that are relatively smooth [8].

To overcome the drawbacks of SPHARM representation, the weighted SPHARM method was proposed by Chung et al. [9], which generalizes SPHARM with additional exponential weights. The exponentially decaying weights substantially reduce the amount of Gibbs phenomenon [6] and allow faster convergence. Despite the global support of the basis functions, it has the potential to conduct local analysis by differential weighting of the SPHARM coefficients [10]. Local shape information can be obtained by evaluating the representation at each fixed point that gives the smoothed version of the coordinates of surfaces [7]. Another approach is to use spherical wavelets (SW) that have compact support at different spatial scales and locations. There have been attempts of using SW for local shape analysis of brain structure, and promising results were reported $[10,11,26]$. The bi-orthogonal spherical wavelet transform [12] that was used suffers from sampling aliasing. Dramatic changes in the wavelet coefficients can be caused by translating an image by even one pixel [13]. More recently in [27] the diffusion wavelet was used for shape representation, which requires training data and a set of landmarks. By using overcomplete SW [14,15], the aliasing problem can be overcome provided there is sufficient sampling at each scale. Moreover, this approach is more robust and sensitive to group differences than bi-orthogonal SW in shape analysis $[16,17]$, and requires no landmarks.

Therefore both weighted SPHARM and SW can be employed for local shape representation. The questions we address here are what is the relationship between them, how to derive SW from weighted SPHARM, how to formulate derived SW for local shape representation, and which one is better in terms of performance and efficiency for a typical biological problem. We first briefly describe the weighted SPHARM method in the next section. Section 3 sheds light on the underlying connections between weighted SPHARM and SW. Based on that, we present a novel derivation of SW and a detailed formulation of wavelet transformation for shape representation in Section 4. In experiments, both the weighted SPHARM and SW are applied for local shape representation of biological structures on different scales, and their performances are compared.

\section{Weighted SPHARM representation}

Given a simple connected 3D object $\mathcal{M}$, spherical parameterization [3] is firstly conducted to create a continuous, uniform mapping from the object surface to the surface of a unit sphere $\Omega$. The result is a bijective mapping between each point $\mathbf{p}$ on $\mathcal{M}$ and a pair of spherical coordinates:

$$
\mathbf{p}(\theta, \varphi)=(x(\theta, \varphi), y(\theta, \varphi), z(\theta, \varphi))^{T}
$$

where the polar angle $\theta \in[0, \pi]$ is the angle between the positive z-axis (north pole) and the vector corresponding to $\mathbf{p}$. The azimuthal angle $\varphi \in[0,2 \pi)$ is the angle between the 
positive $\mathrm{x}$-axis and the projection of $\mathbf{p}$ onto the $\mathrm{x}-\mathrm{y}$ plane. Then shape representation techniques such as weighted SPHARM and SW can be employed to describe the object. Each function of $x(\theta, \varphi), y(\theta, \varphi)$, and $z(\theta, \varphi)$ can be decomposed independently, which is described for $x(\theta, \varphi)$ as an example in the following sections.

Weighted SPHARM is mathematically related to isotropic heat diffusion on $\Omega$ [9], which can be written in the following partial differential equation (PDE) form:

$$
\frac{\partial f}{\partial t}=\Delta_{\Omega} f, f(\theta, \varphi, t=0)=x(\theta, \varphi)
$$

Eq. (2) can be solved by expanding $x$ in SPHARM, which is defined as

$$
Y_{l}^{m}(\theta, \varphi)=\sqrt{\frac{2 l+1}{4 \pi} \frac{(l-m) !}{(l+m) !}} P_{l}^{m}(\cos \theta) e^{i m \varphi}
$$

where $P_{l}^{m}(\mu)=\frac{(-1)^{m}}{2^{l} l !}\left(1+\mu^{2}\right)^{\frac{m}{2}} \frac{d^{l+m}}{d \mu^{l+m}}\left(\mu^{2}-1\right)^{l}$ are the associated Legendre polynomials with the degree $l \geq 0$ and order $|m| \leq l$. The unique solution of Eq. (2) is given by [9]

$$
x(\theta, \varphi)=\sum_{l=0}^{L} \sum_{m=-l}^{l} e^{-l(l+1) t} c_{l x}^{m} Y_{l}^{m}(\theta, \varphi)
$$

where the bandwidth $t$ controls the amount of smoothing, and $c_{l x}^{m}$ are the Fourier coefficients. The implication of Eq. (4) is that the solution decreases exponentially as the bandwidth $t$ increases and smoothes out high spatial frequency noise much faster than low frequency noise, which is the basis of many PDE-based image smoothing methods.

The Fourier coefficients $c_{l x}^{m}$ are estimated by the iterative residual fitting (IRF) method in a hierarchical fashion [9]. Let $L^{2}(\Omega)$ be the space of square integrable functions on the unit sphere $\Omega . L^{2}(\Omega)$ can be decomposed into smaller subspaces as the direct sum of

$$
\text { Spharm }_{0, \ldots, n}=\bigoplus_{l=0}^{n} \text { Spharm }_{l}
$$

which forms a coarse-to-fine hierarchy. The IRF method estimates the $c_{l x}^{m}$ in each subspace $\operatorname{Spharm}_{l}=\operatorname{span}\left\{Y_{l}^{m}\right\}_{m=-l}^{l}$ iteratively by increasing the degree from 0 to $L$.

\section{From weighted SPHARM to SW}

Eq. (4) can be rewritten as

$$
x(p)=\sum_{l=0}^{L} \sum_{m=-l}^{l} e^{-\lambda_{l} t}<x, Y_{l}^{m}(q)>Y_{l}^{m}(p)
$$

where $\lambda_{l}=-l(l+1)$ is the eigenvalue of the Laplace-Beltrami operator $\Delta_{\Omega}$, and $<x, Y_{l}^{m}(q)>$ are the Fourier coefficients $c_{l x}^{m}$. Rearranging the inner product of Eq. (6), weighted SPHARM is casted as the kernel smoothing below 


$$
x(p)=\sum_{l=0}^{L} \sum_{m=-l}^{l} e^{-\lambda_{l} t} Y_{l}^{m}(p) \int_{\Omega} x(q) Y_{l}^{m}(q) d \eta(q)=\int_{\Omega} x(q) K_{t}^{L}(p, q) d \eta(q)
$$

where $d \eta(q)=\sin \theta d \theta d \varphi$, and the symmetric positive kernel $K_{t}^{L}$ is

$$
K_{t}^{L}=\sum_{l=0}^{L} \sum_{m=-l}^{l} e^{-\lambda_{l} t} Y_{l}^{m}(p) Y_{l}^{m}(q)=\sum_{l=0}^{L} e^{-\lambda_{l} t} \sum_{m=-l}^{l} Y_{l}^{m}(p) Y_{l}^{m}(q)
$$

According to the harmonic addition theorem [18], we have

$$
\sum_{m=-l}^{l} Y_{l}^{m}(p) Y_{l}^{m}(q)=\frac{2 l+1}{4 \pi} P_{l}(p \cdot q)
$$

where $P_{l}:[-1,1] \rightarrow \mathbb{R}$ is the Legendre polynomial of degree $l$. Substituting $\lambda_{l}=-l(l+1)$ and Eq. (9) into Eq. (8), we obtain

$$
K_{t}^{L}=\sum_{l=0}^{L} \frac{2 l+1}{4 \pi} e^{-l(l+1) t} P_{l}(p \cdot q)
$$

Eq. (10) is the Gauss-Weierstrass kernel [19].

The term $e^{-l(l+1) t}$ in Eq. (10) can be considered as a discretized version of the continuously defined function $\varphi_{0}(u)=e^{-t u(u+1)}$, which is continuous at 0 , monotonously decreasing and satisfies $\varphi_{0}(0)=1$ [20]. The dilation of $\varphi_{0}$ is given as

$$
\varphi_{j}(u)=D_{j} \varphi_{0}(u)=\varphi_{0}\left(2^{-j} u\right)
$$

where $D_{j}$ is called dilation operator of $\mathrm{j}$-th level. A system of scale discrete scaling function can be generated via $\varphi_{0}$ and its dilations $\varphi_{j}$ as

$$
\Phi_{j}(v)=\sum_{l=0}^{\infty} \frac{2 l+1}{4 \pi} \varphi_{j}(l) P_{l}(v), v \in[-1,1]
$$

The discrete scaling function of Eq. (12) defines a "discrete approximate identity" [20] in $L^{2}(\Omega)$. That is, for any $F \in L^{2}(\Omega)$,

$$
\lim _{j \rightarrow \infty}\left\|F-\Phi_{j} * F\right\|=0
$$

where * designate the convolution operator. The convolution $\Phi_{j} * F$ provides us with a sequence of progressively "sharper" versions of $F$ at different scales, and forms scale spaces $V_{j}$ that satisfy

$$
V_{0} \subset \ldots \subset V_{j} \subset V_{j+1} \subset \ldots L^{2}(\Omega)
$$

Based on the definition of scale discrete scaling functions of Eq. (12), scale discrete wavelets on the sphere can be introduced as the difference of two successive resolution levels

$$
\Psi_{j}(v)=\Phi_{j+1}(v)-\Phi_{j}(v)=\sum_{l=0}^{\infty} \frac{2 l+1}{4 \pi}\left(\varphi_{j+1}(l)-\varphi_{j}(l)\right) P_{l}(v)
$$

Eq. (15) can be considered as a Difference-of-Gaussian (DoG) wavelet. The scale index $\mathrm{j}$ associated to the dyadic value $2^{-j}$ serves as a measure for decreasing frequency 
localization of the scale discrete wavelet kernel. The more levels of wavelet are added, the more accurate representation of the function $F$ can be obtained.

This produces detail spaces $W_{j}$ that contain the information needed to go from an approximation at resolution $\mathrm{j}$ to an approximation at resolution $\mathrm{j}+1$, written as $V_{j+1}=V_{j} \oplus W_{j}$. Besides representing a function by its level-j coarse and level-j detail components, the latter can be broken down to level-(j+1) coarse and detail, and so on. Any function $F \in L^{2}(\Omega)$ can now be represented in the form

$$
V_{J}=V_{0} \oplus W_{0} \oplus W_{1} \oplus \cdots \oplus W_{J-1}
$$

Comparing Eq. (16) to Eq. (5), it is easy to see that the idea behind the IRF method is closely related to the wavelet transform, i.e. representing the function in a coarse-to-fine hierarchy.

\section{$4 \mathrm{SW}$ representation}

The SW derived in Section 3 exploit the geometry of the sphere that encode in the system of spherical harmonics. Their analyzing functions are poorly localized, and in fact they do not really resemble wavelets [21]. The wavelet dilation is explicitly defined in harmonic space on $\Omega$, which lacks some important properties [22]. First, the corresponding properties of a radial and conformal diffeomorphism is lost, as the harmonic dilation does not act on points. Second, the evolution in real space of the localization and directionality properties of functions on the sphere through harmonic dilation is not known analytically due to its explicit definition in harmonic space. In the following sections, we propose a new way to construct over-complete SW based on the group theoretic approach [21], and use the theoretical results from the work of [17] to build self-invertible filter banks, which are employed for decomposing and reconstructing images.

\subsection{Constructing over-complete SW}

In the group theoretic approach, over-complete wavelets on the sphere are built as inverse stereographic projections of wavelets on the plane. The stereographic projection is the unique radial conformal diffeomorphism mapping the sphere $\Omega$ onto the plane $\mathbb{R}^{2}$ [21], and SW are constructed by projecting admissible Euclidean planar wavelets on to the sphere by

$$
\psi_{\Omega}(\theta, \varphi)=\left(1+\tan ^{2} \frac{\theta}{2}\right) \psi_{\mathbb{R}^{2}}(r, \varphi)
$$

where $r(\theta)=2 \tan (\theta / 2)$ is the polar coordinate in the plane tangent to the sphere at the north pole. The term $1+\tan ^{2}(\theta / 2)$ is to ensure the unitarity of the projection.

Different from Eq. (15), we construct the spherical DoG wavelet by projecting its Euclidean planar formula on to the sphere. DoG is a rotation invariant wavelet, and has the following form on the plane

$$
\operatorname{DoG}(x, y)=\frac{1}{2 \pi}\left(\frac{1}{\sigma_{1}^{2}} e^{-\left(x^{2}+y^{2}\right) / 2 \sigma_{1}^{2}}-\frac{1}{\sigma_{2}^{2}} e^{-\left(x^{2}+y^{2}\right) / 2 \sigma_{2}^{2}}\right)
$$


Its inverse stereographic projection defines the DoG wavelet on the sphere as

$$
\operatorname{DoG}(\theta, \varphi)=\frac{1}{2 \pi}\left(1+\tan ^{2}(\theta / 2)\right)\left(\frac{1}{\sigma_{1}^{2}} e^{-\frac{2}{\sigma_{1}^{2} \tan ^{2} \frac{\theta}{2}}}-\frac{1}{\sigma_{2}^{2}} e^{-\frac{2}{\sigma_{2}^{2}} \tan ^{2} \frac{\theta}{2}}\right)
$$

which is independent of the azimuthal angle $\varphi$ and is said to be zonal or axisymmetric.

\subsection{Constructing self-invertible filter banks}

It is desirable to employ identical filters for both analysis and synthesis in the wavelet domain, which follows the intuitive notion that a convolution coefficient corresponds to the contribution of the corresponding filter to the reconstructed signal [17]. Otherwise, the effects of nonlinear processing of wavelet coefficients could propagate to spatial locations and frequencies other than those which were used to compute the coefficients [13]. We constructed self-invertible filter banks according to the work of [17].

The $\mathrm{n}^{\text {th }}$ analysis filters $\tilde{h}_{n}$ are the stereographic dilation [21] of Eq. (19)

$$
\tilde{h}_{n}(\theta, \varphi)=\left(\prod_{i=1}^{n} b_{i}\right) D_{a_{n}} \operatorname{DoG}(\theta, \varphi)
$$

where $b_{i}$ are the amplitude scaling parameters that control the tradeoff between selfinvertibility and norm-preserving dilation. The stereographic dilation operator $D_{a_{n}}$ on $G \in L^{2}(\Omega)$, for a continuous dilation factor $a \in R_{+}^{*}$, is defined as

$$
\left[D_{a} G\right](\theta, \varphi)=\lambda^{1 / 2}(a, \theta) G\left(2 \arctan \left(\frac{1}{a} \tan \frac{\theta}{2}\right), \varphi\right)
$$

where the normalization factor $\lambda^{1 / 2}(a, \theta)$ is uniquely determined by the requirement that the dilation of functions in $L^{2}(\Omega)$ must be a unitary operator, i.e. preserve the scalar product, and specifically the norm of functions. For self-invertible filter banks, the synthesis filter $h_{n}$ is the same as the analysis filters $\tilde{h}_{n}$.

\subsection{Spherical wavelet transform}

A wavelet basis on the sphere may now be constructed by rotations of $\tilde{h}_{n}$. The rotation operator $R$ is parameterized in terms of the three Euler angles $(\alpha, \beta$, and $\gamma)$. The three angles specify an element of the rotation group $S O(3)$. The effects of rotation on the SPHARM coefficients of a square-integrable function on $\Omega$ is expressible in terms of Wigner-D functions $D_{l}^{m n^{\prime}}(\alpha, \beta, \gamma)$ [23]. The rotation transforms each SPHARM of degree $l$ into a linear combination of SPHARM of the same degree but possibly different orders [17]. That is

$$
[R(\alpha, \beta, \gamma) G]_{l}^{m}=\sum_{m^{\prime}=-l}^{l} D_{l}^{m m^{\prime}}(\alpha, \beta, \gamma) G_{l}^{m^{\prime}}
$$


The corresponding wavelet family $R(\alpha, \beta, \gamma) \tilde{h}_{n}(\theta, \varphi)$ provides an over-complete set of functions in $L^{2}(\Omega)$. A spherical continuous wavelet transform (SCWT) of $x(\theta, \varphi)$ can be given in terms of a wavelet basis by the projection on to each wavelet basis function by spherical convolution

$$
W_{n}(\alpha, \beta, \gamma)=\int_{\Omega}\left[R(\alpha, \beta, \gamma) \tilde{h}_{n}\right] *(\theta, \varphi) x(\theta, \varphi) d \Omega
$$

where $*$ denotes complex conjugation. It is worth to note that the spherical harmonic coefficients are zero for orders other than 0 due to the axisymmetry of DoG wavelets. The fast spherical convolution [24] can be used to compute Eq. (23).

To produce reconstructed surface components, the synthesis filters are used to project a function in $L^{2}(S O(3))$ onto $L^{2}(\Omega)$ by inverse convolution

$$
\hat{x}_{n}(\theta, \varphi)=\int_{S O(3)}\left[R(\alpha, \beta, \gamma) h_{n}\right](\theta, \varphi) W_{n}(\alpha, \beta, \gamma) d \rho
$$

where the integration is over the Euler angles $d \rho=\sin \beta d \alpha d \beta d \gamma$.

The reconstructed surface is obtained by summing over the response of all filter pairs

$$
\hat{x}(\theta, \varphi)=\sum_{n} \int_{S O(3)}\left[R(\alpha, \beta, \gamma) h_{n}\right](\theta, \varphi) W_{n}(\alpha, \beta, \gamma) d \rho
$$

The SCWT is defined continuously in above formulation. In practice, effective analysis requires discretization. We sample the output of the continuous convolution $W_{n}(\alpha, \beta, \gamma)$ and the surface reconstruction of Eq. (25) is approximated by finite weighted summations. For axisymmetric analysis/synthesis filter banks of a finite SPHARM degree, latitudelongitude sampling guarantees that the filter bank has the same frequency response under the continuous and discrete convolution [17].

\section{Experimental results}

We use weighted SPHARM and over-complete SW for shape representation of biological structures, including both macro and micro scale images. The macro scale images are amygdala images published in [7], which consist of manual amygdala segmentations of MRI data of 10 control and 12 autistic subjects. The micro scale images are ten stacks of neutrophil cell images, which are labeled with cell mask orange dye to stain the plasma membrane, and are imaged by spinning disk confocal microscopy with $\mathrm{Z}$ stacking. A semiautomatic method was employed for cell segmentation, which was further processed to ensure that interior holes were filled and isolated islands removed. Thus, a binary segmentation volume is created whose voxel surface has a spherical topology. The typical amygdala image has 2302 vertices and 4600 faces, which is much smaller than the size of a cell image with 24538 vertices and 49072 faces. Moreover, cell images have more complex and finer structures than amygdala images.

Both shape representation techniques require continuous spherical parameterization to map from the surface mesh to a unit sphere. The spherical parameterization method presented by Brechbühler et al. (1995) is applied, and its implementation in the shape analysis toolbox of SPHARM-PDM [25] was used, where PDM stands for Point Distribution Models. 
The root mean-squared error (RMSE) was calculated for quantitatively comparing the results provided by different representation methods. The RMSE is computed by

$$
R M S E=\sqrt{\frac{\sum_{i=1}^{N}\left(\mathcal{M}\left(\mathbf{p}_{i}\right)-\tilde{\mathcal{M}}\left(\mathbf{p}_{i}\right)\right)^{2}}{N}}
$$

where $\mathcal{M}$ indicates a true object surface and $\tilde{\mathcal{M}}$ its representation, which is evaluated at $\mathrm{N}$ sampling points $\left\{\mathbf{p}_{i}\right\}_{i=1}^{N}$. The average errors and computational times for amygdala and neutrophil cell images are reported separately for each method in Tables 1-4.

The weighted SPHARM code was obtained from the author's website. The bandwidth $t$ in Eq. (4) that controls the dispersion of the kernel is set to 0.001. Another issue that needs to be addressed is the degree. It is important to find the optimal degree where the goodness of representation and the computational cost are balanced. Chung et al. [9] presented a statistical framework for automatically determining the optimal degree that does not depend on the size of object. They reported that the optimal degree is 42 for bandwidth $\mathrm{t}=0.001$, which is used for weighted SPHARM representations. We also present results with degree 10,20, and 30. For the reason of comparison, we additionally report the result with setting the bandwidth to $t=0.0001$ and the optimal degree to 78 [9]. As the degree increases over 78, the RMSE flattens out, and the decrease in error is no longer significant.

SW decompose the image into component signals at different scales. Each kernel at different scale acts as a bandpass filter. Since the DoG wavelet of Eq. (19) is axisymmetric, the spherical harmonic coefficients are zero for orders other than 0 . We choose the first four hundred spherical harmonics of order 0 as the set of basis functions, and define the set of scales to be $a=\left\{2^{-n}\right\}, n=-1,0, \ldots, 5$ with $n=0$ corresponding to the undilated template. The spherical harmonic coefficients are precomputed, and stored on a hard drive. The amplitude scaling parameters $b_{i}$ in Eq. (20) are set to be the same for all scales, which results in the frequency response of filters having comparable amplitude at different scales [17]. The sigma values in Eq. (19) are set as $\sigma_{1}=0.625$ and $\sigma_{2}=1$, which makes it a good substitute for the Laplacian of Gaussian.

Fig. 1 shows shape representations of an example surface of left amygdala via both weighted SPHARM and SW. It can be seen that 20 degree weighted SPHARM and level 1 and $3 \mathrm{SW}$ representations are not good enough to describe the local shape. The weighted SPHARM of 42 and 78 degrees and level 5 and $7 \mathrm{SW}$ representations are visually very close to the original surface of Fig. 1(a). That is confirmed by the RMSE values listed in Tables 1 and 2. The 42 degree weighted SPHARM representation has the same RMSE value of 0.16 as the level $5 \mathrm{SW}$ representation. In terms of computation time, weighted SPHARM (1.3 s) is much faster than SW (29.13 s) for representing small size amygdala. For a simple (no complex and fine structures) biological object with small size, weighted SPHARM should be a better choice that can achieve considerably good results efficiently. However, further increasing the degree of weighted SPHARM has less effect than increasing the level of SW. Weighted SPHARM representation with 78 degree can only achieve a RMSE value of 0.07 , which is higher than the level $6 \mathrm{SW}$ representation with a RMSE value of 0.04 .

The better performance of SW is demonstrated when representing cell images as shown in Fig. 2. Comparing the shape representation with level $7 \mathrm{SW}$ to the original surface, they 


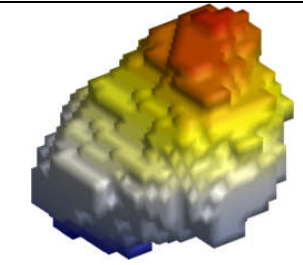

(a)

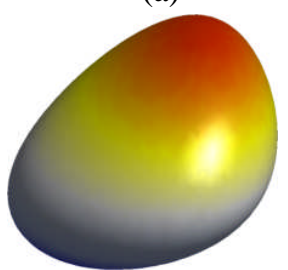

(e)

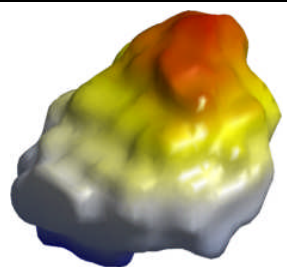

(b)

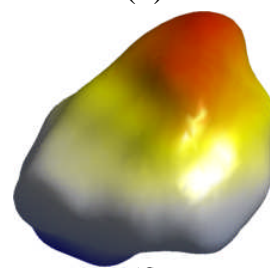

(f)

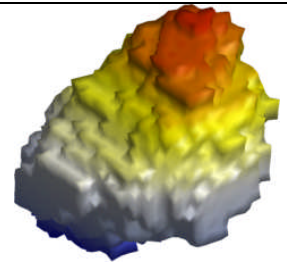

(c)

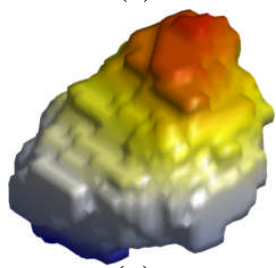

(g)

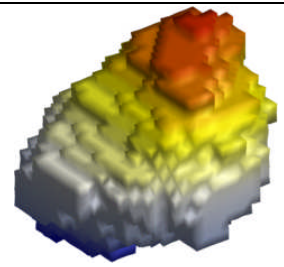

(d)

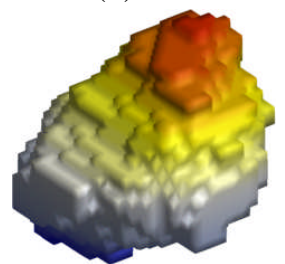

(h)

Fig. 1 (a) An example surface of left amygdala. (b-d) Its corresponding 20, 42, and 78 degrees weighted SPHARM representation. (e-h) Its level 1, 3, 5, and $7 \mathrm{SW}$ representation.

Table 1. The average RMSE values and the average running times of weighted SPHARM for amygdala representation.

\begin{tabular}{cccccc}
\hline Degrees & $\mathbf{1 0}$ & $\mathbf{2 0}$ & $\mathbf{3 0}$ & $\mathbf{4 2}$ & $\mathbf{7 8}$ \\
\hline RMSE & 0.41 & 0.33 & 0.26 & 0.16 & 0.07 \\
Time (s) & 0.1 & 0.32 & 0.66 & 1.3 & 5.46 \\
\hline
\end{tabular}

Table 3. The average RMSE values and the average running times of weighted SPHARM for cell representation.

\begin{tabular}{cccccc}
\hline Degrees & $\mathbf{1 0}$ & $\mathbf{2 0}$ & $\mathbf{3 0}$ & $\mathbf{4 2}$ & $\mathbf{7 8}$ \\
\hline RMSE & 0.81 & 0.53 & 0.44 & 0.39 & 0.31
\end{tabular}

Time (s) $0.923 .19 \quad 7.58 \quad 15.57 \quad 57.51$

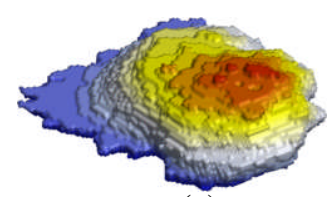

(a)

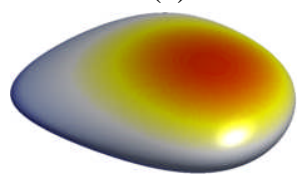

(e)

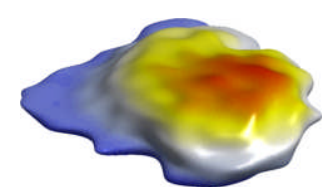

(b)

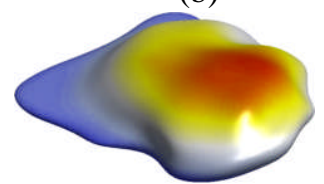

(f)

Table 2. The average RMSE values and the average running times of SW for amygdala representation.

\begin{tabular}{|c|c|c|c|c|c|c|c|}
\hline Levels & 1 & 2 & 3 & 4 & 5 & 6 & 7 \\
\hline RMS & 7.57 & 0.53 & 0.39 & 0.3 & 0.16 & 0.04 & 0.02 \\
\hline
\end{tabular}

Time (s) $10.47 \quad 15.08 \quad 19.7824 .4129 .13 \quad 33.7538 .4$

Table 4. The average RMSE values and the average running times of $\mathrm{SW}$ for cell representation.

\begin{tabular}{cccccccc}
\hline Levels & $\mathbf{1}$ & $\mathbf{2}$ & $\mathbf{3}$ & $\mathbf{4}$ & $\mathbf{5}$ & $\mathbf{6}$ & $\mathbf{7}$ \\
\hline RMSE & 5.08 & 1.25 & 0.72 & 0.48 & 0.37 & 0.16 & 0.08
\end{tabular}

Time (s) 10.8615 .5420 .2524 .9129 .5634 .2638 .92

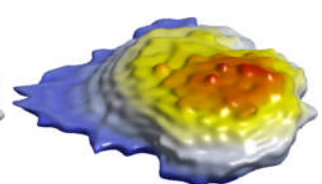

(c)

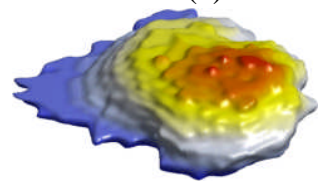

(g)

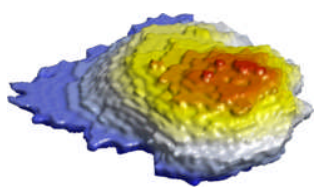

(d)

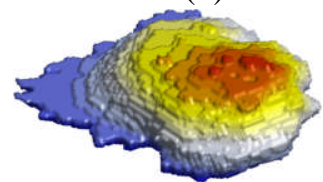

(h)

Fig. 2 (a) An example surface of a neutrophil cell. (b-d) 20, 42, and 78 degrees weighted SPHARM representation of (a). (e-h) Level 1, 3, 5, and $7 \mathrm{SW}$ representation of (a). 
are visually almost undistinguishable. The RMSE values of SW with levels 6 and 7 are 0.16 and 0.08 , respectively, which are better than 0.31 for the weighted SPHARM representation with degree 78 (see Tables 3 and 4). The limitation of weighted SPHARM is that it fixes the bandwidth for all the components appearing on the surface. It has a limited ability to detect subtle changes. By using multiscale bandwidths, SW are able to represent very fine structures. The total running time is higher for degree 78 weighted SPHARM (57.51 s) than level $7 \mathrm{SW}(38.92 \mathrm{~s})$. Comparing Table 2 to 4 , it is interesting to note that the object size has negligible influence on the running time of $\mathrm{SW}$, as it increased only from $38.4 \mathrm{~s}$ to $38.92 \mathrm{~s}$ using level $7 \mathrm{SW}$ for representing amygdala and cell images, respectively. In contrast, the total running time of degree 78 weighted SPHARM increased dramatically, from $5.46 \mathrm{~s}$ to $57.51 \mathrm{~s}$. Therefore, SW should be a better choice for representing large and complex biological structures.

\section{Conclusions}

There is a close relationship between weighted SPHARM and SW. From the weighted SPHARM formulation, DoG SW can be derived. The idea behind the IRF method used for weighted SPHARM coefficients estimation is similar to the wavelet transform, i.e. representing the function in a coarse-to-fine hierarchy. Both the reformulated SW and weighted SPHARM can be used for local shape representation of biological structures. For more complex and larger objects, SW should be the better choice which can achieve better results more efficiently. For small size objects without fine structures, weighted SPHARM yield a representation of similar quality as with SW, but are faster to compute.

\section{Acknowledgement}

This work was supported by the Biotechnology and Biological Sciences Research Council (grant number: BBI0082091).

\section{References}

[1] F. L. Bookstein. Morphometric Tools for Landmark Data. Cambridge University Press, 2003.

[2] H. Blum. Biological shape and visual science. J. Theor. Biol. 38 (2), 205-287, 1973.

[3] C. Brechbuhler, G. Gerig, and O. Kubler. Parameterization of closed surfaces for 3D shape description. Comp. Vis. Image Understanding, 61:154-170, 1995.

[4] R. Schudy, and D. Ballard. Towards an anatomical model of heart motion as seen in 4-D cardiac ultrasound data. The 6th Conference on Computer Applications in Radiology and ComputerAided Analysis of Radiological Images, 1979.

[5] L. Shen, H. Farid, and M. A. McPeek. Modeling 3-dimensional morphological structures using spherical harmonics. Evolution, 63(4), 1003-1016, 2009.

[6] A. Gelb. The resolution of the Gibbs phenomenon for spherical harmonics. Math. Comput. 66, 699-717, 1997.

[7] M. K. Chung, K. J. Worsley, B. M. Nacewicz, K. M. Dalton, and R. J. Davidson. General multivariate linear modeling of surface shapes using SurfStat. NeuroImage, 53, 491-505, 2010. 
[8] L. Shen, S. Kim, and A. J. Saykin. Fourier method for large scale surface modeling and registration. Computers and Graphics (Special Issue on Shape Modeling International 2009), 33(3):299-311, 2009.

[9] M. K. Chung, K. M. Dalton, L. Shen, A. C. Evans, and R. J. Davidson. Weighted Fourier series representation and its application to quantifying the amount of gray matter. Special Issue of IEEE Transactions on Medical Imaging, on Computational Neuroanatomy, 26, 566-581, 2007.

[10] P. Yu, P. Grant, Y. Qi, X. Han, F. Segonne, R. Pienaar, E. Busa, J. Pacheco, N. Makris, and R. Buckner, et al. Cortical surface shape analysis based on spherical wavelets. IEEE Transactions on Medical Imaging, 26(4), 582-598, 2007.

[11] D. Nain, M. Styner, M. Niethammer, J. Levitt, M. Shenton, G. Gerig, A. Bobick, and A. Tannenbaum. Statistical shape analysis of brain structures using spherical wavelets. IEEE Symposium on Biomedical Imaging ISBI, 2007.

[12] P. Schröder, and W. Sweldens. Spherical wavelets: Efficiently representing functions on the sphere. Computer Graphics Proceedings (SIGGRAPH 95), 161-172, 1995.

[13] E. P. Simoncelli, W. T. Freeman, E. H. Adelson, and D. J. Heeger. Shiftable Multi-scale Transforms. IEEE Transaction Information Theory, 38(2), 587-607, 1992.

[14] I. Bogdanova, P. Vandergheynst, J. Antoine, L. Jacques, and M. Morvidone. Stereographic Wavelet Frames on the Sphere. Applied and Computational Harmonic Analysis, 19, 223-252, 2005.

[15] B.T.T. Yeo, P. Yu, P.E. Grant, B. Fischl, P. Golland. Shape analysis with overcomplete spherical wavelets. Proceedings of the International Conference on Medical Image Computing and Computer Assisted Intervention (MICCAI), 5241 of LNCS, 468--476, 2008.

[16] P. Yu, B.T.T. Yeo, P.E. Grant, B. Fischl, P. Golland. Cortical Folding Development Study based on Over-complete Spherical Wavelets. In: MMBIA, 2007.

[17] B.T.T. Yeo, W. Ou, and P. Golland. On the Construction of Invertible Filter Banks on the 2Sphere. IEEE Transactions on Image Processing 17(3), 283-300, 2008.

[18] H. Groemer. Geometric Applications of Fourier Series and Spherical Harmonics. Cambridge, U.K.: Cambridge Univ. Press, 1996.

[19] W. Freeden, M. Schreiner, and R. Franke. A survey on spherical spline approximation. Surv. Math. Ind., 7, 29-85, 1997.

[20] W. Freeden, and M. Schreiner. Orthogonal and non-orthogonal multiresolution analysis, scale discrete and exact fully discrete wavelet transform on the sphere. Constr. Approx., 14, 493-515, 1998.

[21] J.P. Antoine, and P. Vandergheynst. Wavelets on the 2-sphere: A group-theoretical approach. Appl. Comput. Harmon. Anal., 7(3), 262-291, 1999.

[22] P. Vandergheynst and Y. Wiaux. Wavelets on the Sphere. In "Four Short Courses on Harmonic Analysis: Wavelets, Frames, Time-Frequency Methods, and Applications to Signal and Image Analysis" (Eds. Brigitte Forster, Peter Massopust, Brigitte Forster-Heinlein), Boston, MA : Birkhäuser Boston 2010.

[23] J.J. Sakurai. Modern Quantum Mechanics, 2nd ed. Reading, MA: Addison Wesley, 1994.

[24] J.R. Driscoll and D.M. Healy. Computing Fourier transforms and convolutions on the 2-Sphere. Adv. Appl. Math., 15, 202-250, 1994. 
[25] M. Styner, I. Oguz, S. Xu, C. Brechbuhler, D. Pantazis, J. Levitt, M. E. Shenton, and G. Gerig. Framework for the statistical shape analysis of brain structures using spharm-pdm. Insight J., 1071, 242-250, 2006.

[26] D. Nain, S. Haker, A. Bobick, and A. Tannenbaum. Multiscale 3-d shape representation and segmentation using spherical wavelets. IEEE Trans Med Imaging, 26(4):598-618, 2007.

[27] S. Essafi, G. Langs \& N. Paragios. Hierarchical 3D Diffusion Wavelets Shape Priors. IEEE International Conference in Computer Vision (ICCV), 2009. 